\title{
Um Panorama das Pesquisas Científicas focado no ENCompIF: Um Mapeamento Sistemático
}

\author{
Filipe Arantes Fernandes, Otacílio José Pereira, Loham Santos da Silva
}

\author{
Instituto Federal de Educação, Ciência e Tecnologia do Sudeste de Minas Gerais (IF \\ Sudeste MG) \\ Caixa Postal 413 - 36.909-300 - Manhuaçu - MG - Brasil \\ \{filipe.arantes, otacilio.pereira, loham.silva\}@ifsudestemg.edu.br
}

\begin{abstract}
The goal of this work is to show a Systematic Mapping (SM) of papers published in the National Computing Meeting of Federal Institutes (ENCompIF), with the purpose of characterizing the state of the art of computing researches carried out by institutions of the Federal Education Network. It was defined as a research strategy, primary studies published in ENCompIF in the period between 2013 and 2019 and it was found that, despite the slight decline in publications over the years, a community has intensified the involvement of students, as well as in the development of solutions for support the teaching and learning process through computational solutions.
\end{abstract}

Resumo. O objetivo deste trabalho é apresentar um Mapeamento Sistemático (MS) de artigos publicados no Encontro Nacional de Computação dos Institutos Federais (ENCompIF), com o propósito de caracterizar o estado da arte de pesquisas em computação realizadas por instituições da Rede Federal de ensino. Definiu-se como estratégia de busca, estudos primários publicados no ENCompIF no período entre 2013 e 2019 e verificou-se que apesar do leve declínio de publicações ao longo dos anos, a comunidade tem intensificado esforços no envolvimento dos discentes, bem como no desenvolvimento de soluções para apoiar o processo de ensino e aprendizagem por meio de soluções computacionais.

\section{Introdução}

Desde a sua semente, a partir do conjunto de escolas de Artífices e Aprendizes em 1909, a Rede Federal de Educação Profissional, Científica e Tecnológica desempenha papel relevante para que todas as pessoas tenham acesso às conquistas científicas e tecnológicas do país [MEC, 2020]. Ainda de acordo com o MEC [2020], após a entrega de 208 (duzentos e oito) novas unidades entre 2011 e 2014, a Rede Federal de ensino atingiu a marca de 562 (quinhentos e sessenta e duas) unidades e vale destacar, com extrema capilaridade, atingindo regiões urbanas e sobretudo rurais do nosso território. Portanto, munir esta rede com instrumentos que permitam conhecer sua atuação e produção fortalece a sua identidade e vocação científica, sobretudo na pesquisa aplicada.

Compreender a atuação de certa comunidade científica permite que seus integrantes organizem suas tomadas de decisão sobre quais ações e direções adotar em certo contexto. No âmbito da pesquisa, mapear a produção de uma rede de instituições permite identificar trabalhos interessantes e, a partir disso, replicar e abrir novas frentes em outras regiões, avaliar interesses comuns para o estabelecimento de parcerias e ainda, perceber espaços vazios a explorar com novas linhas de investigação. Importante ainda salientar que conhecer sobre trabalhos de computação, uma área sensível para nortear o futuro da educação e do 
emprego, revisões da literatura podem contribuir para discutir o rumo dos avanços tecnológicos para todos, aspecto chave na missão da Rede Federal. Outros estudos foram realizados com o propósito de analisar contribuições e/ou oportunidades de pesquisas futuras na área de Computação [Araujo et al., 2017; Souza et al., 2016], porém nenhum focou em pesquisas realizadas especificamente na Rede Federal de Ensino.

Ainda no âmbito da pesquisa, a Rede Federal conta com o Encontro Nacional de Computação dos Institutos Federais (ENCompIF), evento satélite do Congresso da Sociedade Brasileira de Computação (CSBC), no qual iniciou-se em 2013 e tem sido realizado anualmente, excluindo-se o ano de 2015. Em sua $1^{\mathrm{a}}$ edição, a cidade de Maceió-AL deu início às atividades do evento, tendo sua $2^{\mathrm{a}}$ edição em Brasília (2014), $3^{\mathrm{a}}$ edição em Porto AlegreRS (2016), $4^{\mathrm{a}}$ edição em São Paulo-SP (2017), $5^{\mathrm{a}}$ edição em Natal-RN e sua $6^{\mathrm{a}}$ edição em Belém-PA (2019). Sua principal missão é reunir professores e alunos dos Institutos Federais (IFs) e outras instituições que oferecem cursos técnicos de informática para que suas pesquisas realizadas possam ser apresentadas.

Desta forma, o objetivo deste trabalho é apresentar um Mapeamento Sistemático (MS) de trabalhos publicados no ENCompIF, com o propósito de caracterizar o estado da arte de pesquisas em computação realizadas por instituições da Rede Federal de ensino. $\mathrm{O}$ MS da literatura oferece uma visão geral da área de estudo, quantificando os resultados. Assim, é possível identificar tendências e lacunas de pesquisas a serem estudadas [Silva et al., 2019].

As próprias questões da sistematização da pesquisa, suas questões e parâmetros de classificação, juntamente com o levantamento dos trabalhos do evento, são tarefas que buscam oferecer um mapa útil para que docentes e corpo diretivo posicionem-se no contexto das instituições da Rede Federal. Além disso, espera-se que este trabalho possa fomentar reflexões estimuladas pelo evento científico em questão, bem como permitir a integração e parcerias entre IFs, Universidades Federais (UFs), iniciativas privadas, dentre outros.

O presente artigo está organizado da seguinte forma: a Seção 2 apresenta a forma de condução do MS com suas questões de pesquisa e parâmetros de seleção e exclusão de artigos. Na sequência, a Seção 3 apresenta os resultados e análises e, por fim, a Seção 4 apresenta as considerações finais e trabalhos futuros.

\section{Planejamento do Mapeamento Sistemático}

O objetivo deste estudo secundário é caracterizar o estado da arte de pesquisas científicas em computação, analisando estudos primários publicados no ENCompIF, e assim obter um panorama das pesquisas desenvolvidas nas instituições da Rede Federal, bem como identificar possíveis lacunas e oportunidades para pesquisas futuras. A principal questão de pesquisa que norteia este estudo é: Qual o estado da arte de pesquisas em computação desenvolvidas nas instituições da Rede Federal?

O planejamento e execução deste Mapeamento Sistemático (MS) foi baseado nas diretrizes apresentadas em [Kitchenham et al. 2011; Petersen et al. 2008]. Nesta seção é apresentada as etapas do planejamento deste estudo secundário.

\subsection{Questões de Pesquisa}

As questões de pesquisa definidas neste MS visam fornecer informações específicas sobre os aspectos relevantes das pesquisas científicas em computação desenvolvidas nas instituições 
da Rede Federal, com enfoque em artigos publicados no ENCompIF. Isso inclui obter evidências quanto a evolução histórica das publicações, identificar as instituições da Rede Federal mais atuantes, bem como identificar as principais contribuições realizadas e classificá-las em áreas da computação. Neste sentido, as questões de pesquisa que conduzem este estudo secundário são apresentadas a seguir:

QP1: Qual a evolução da quantidade de pesquisas publicadas no ENCompIF?

QP2: Quais são os IFs e as regiões atuantes no ENCompIF?

QP3: Quais são as contribuições realizadas pelos estudos?

QP4: Os estudos atuam em quais áreas da computação?

\subsection{Estratégia de Busca}

Como o foco deste estudo secundário é obter evidências sobre pesquisas de computação desenvolvidas especificamente na Rede Federal de ensino, definiu-se como estratégia de busca deste MS, estudos primários publicados no ENCompIF no período entre 2013 e 2019. Considerando que o principal objetivo do MS é obter um panorama sobre pesquisas em computação desenvolvidas na Rede Federal de ensino, bem como no ENCompIF como principal evento da área, não foi estabelecida uma string de busca, ou seja, a busca por termos-chave nos diferentes campos das questões de pesquisa, como a maioria dos estudos secundários definem. O processo de busca das publicações se deu no acesso dos anais de cada ano, através da plataforma SBC OpenLib ${ }^{1}$, pelos pesquisadores envolvidos no MS. Os critérios de inclusão de publicações para este estudo são: (i) os artigos devem ser primários; (ii) a versão completa dos artigos deve estar disponível na Web; (iii) os artigos devem contemplar alguma área da computação e (iv) pelo menos um autor do artigo deve pertencer a alguma instituição da Rede Federal de Ensino.

Os critérios de exclusão consistem na negação ou complemento dos critérios de inclusão definidos, bem como na verificação de artigos que são evolução de trabalhos anteriores. Nestes casos, deve ser considerada a publicação mais recente.

\section{Resultados e Análises}

Esta seção apresenta as respostas das questões de pesquisa definidas na subseção 2.1. Nas seis edições do ENCompIF foram publicados 112 estudos. Ao aplicar os critérios de inclusão e exclusão, todos os estudos foram selecionados para análise.

\subsection{QP1: Qual a evolução da quantidade de pesquisas publicadas no ENCompIF?}

O ENCompIF é um evento satélite do Congresso da Sociedade Brasileira de Computação (CSBC), no qual iniciou-se em 2013 e tem sido realizado anualmente, excluindo-se o ano de 2015. O propósito desta questão de pesquisa é identificar como tem sido a evolução da quantidade de publicações no evento. Como pode ser observado no Gráfico 1, a partir do primeiro evento, a quantidade de publicações tem sofrido queda razoável.

Vários motivos podem ser especulados como razão do declínio das publicações ao longo dos anos, como por exemplo, dificuldades no custeio de diárias dos pesquisadores pelos Institutos Federais e/ou agências de fomento, interesse dos pesquisadores em divulgar seus trabalhos em outros eventos, melhoria nos critérios de revisão dos estudos, dentre outros.

\footnotetext{
${ }^{1}$ https://sol.sbc.org.br/
} 


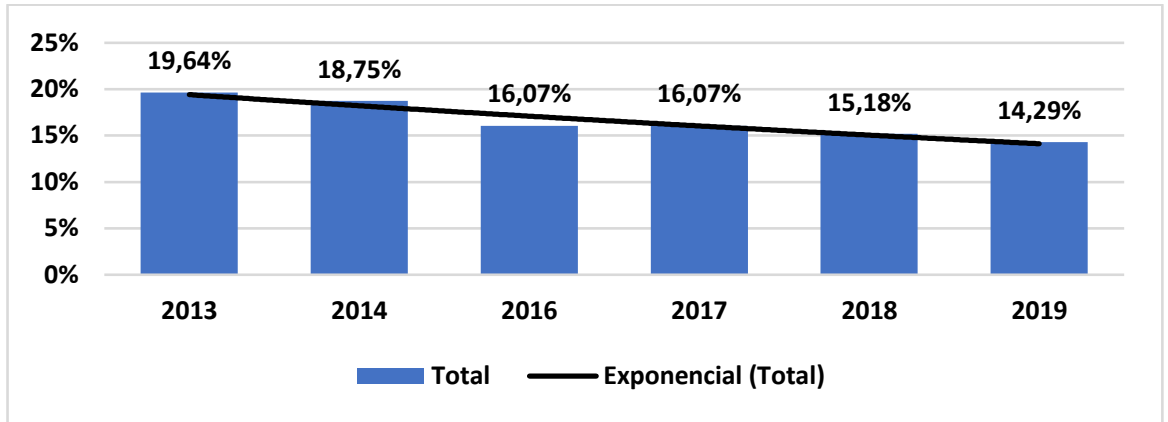

Gráfico 1. Frequência de publicação nas edições do ENCompIF (Fonte: autor)

\subsection{QP2: Quais são os IFs atuantes no ENCompIF?}

Sendo o ENCompIF como o principal encontro de computação dos IFs, esta questão de pesquisa visa como resposta, identificar os IFs mais atuantes no evento ao longo dos anos. $\mathrm{O}$ Gráfico 2 apresenta a frequência de publicações ao longo dos anos por IF. Como pode ser observado, o IFBA ${ }^{2}$ (IF da Bahia), IFMS $^{3}$ (IF de Mato Grosso do Sul), IFC ${ }^{4}$ (IF Catarinense), $\operatorname{IFPB}^{5}$ (IF da Paraíba) e IFSP ${ }^{6}$ (IF de São Paulo) são as instituições que se destacam no evento.

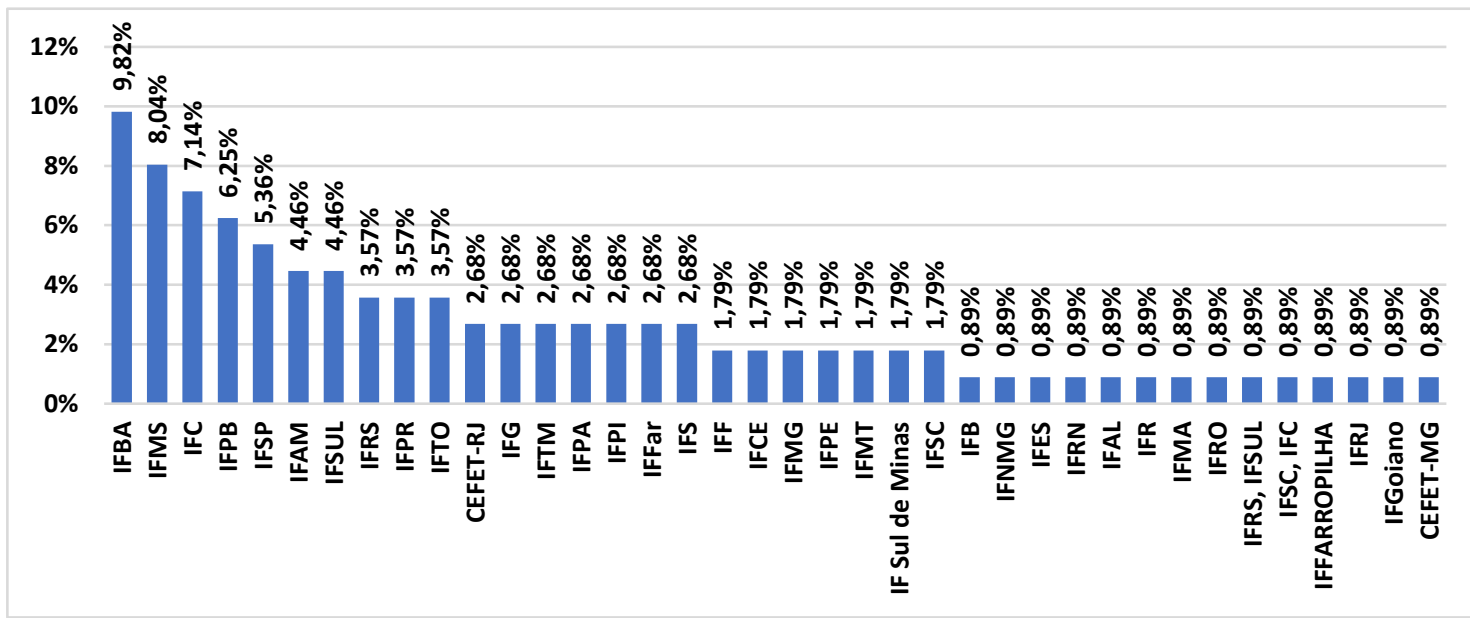

Gráfico 2. Frequência de publicações por IF (Fonte: autor)

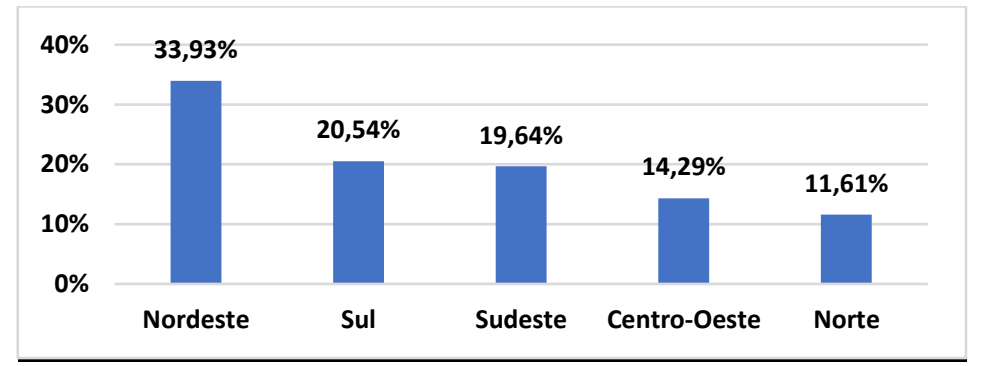

Gráfico 3. Frequência de publicações por Região (Fonte: autor)

\footnotetext{
2 https://portal.ifba.edu.br/

${ }^{3}$ https://www.ifms.edu.br/

${ }^{4}$ http://ifc.edu.br/

5 https://www.ifpb.edu.br/

${ }^{6}$ https://www.ifsp.edu.br/
} 
O Gráfico 3 apresenta a frequência de publicações por Região. Como pode ser observado, a região Nordeste se destaca seguida das regiões Sul e Sudeste com participação equiparável de publicações seguidas das regiões Centro-Oeste e Norte.

\subsection{QP3: Quais são as contribuições realizadas pelos estudos?}

O Gráfico 4 apresenta a resposta da questão de pesquisa definida, cujo objetivo é identificar quais são os tipos de contribuições realizadas pelos pesquisadores. A lista de classificação emergiu durante a leitura dos artigos. Considerando a área de computação, grande parte dos estudos relataram o desenvolvimento de software com 52 (cinquenta e dois) estudos e de sistemas computacionais com (12) doze estudos com aplicabilidade em diversas áreas, tais como apoio ao ensino, saúde, gerenciamento de projetos, monitoramento de redes, jogos educacionais, dentre outros. Considerou-se como sistemas computacionais os trabalhos que integram hardware e software como proposta de solução.

Outra contribuição que se destaca é o uso de recursos computacionais para apoiar pessoas com deficiência, classificado neste MS como tecnologias assistivas. Os estudos desta natureza contribuíram no apoio ao ensino de programação para surdos, desenvolvimento cognitivo em pessoas com Alzheimer, pessoas com problemas de mobilidade, ensino de matemática para surdos, dentre outros.

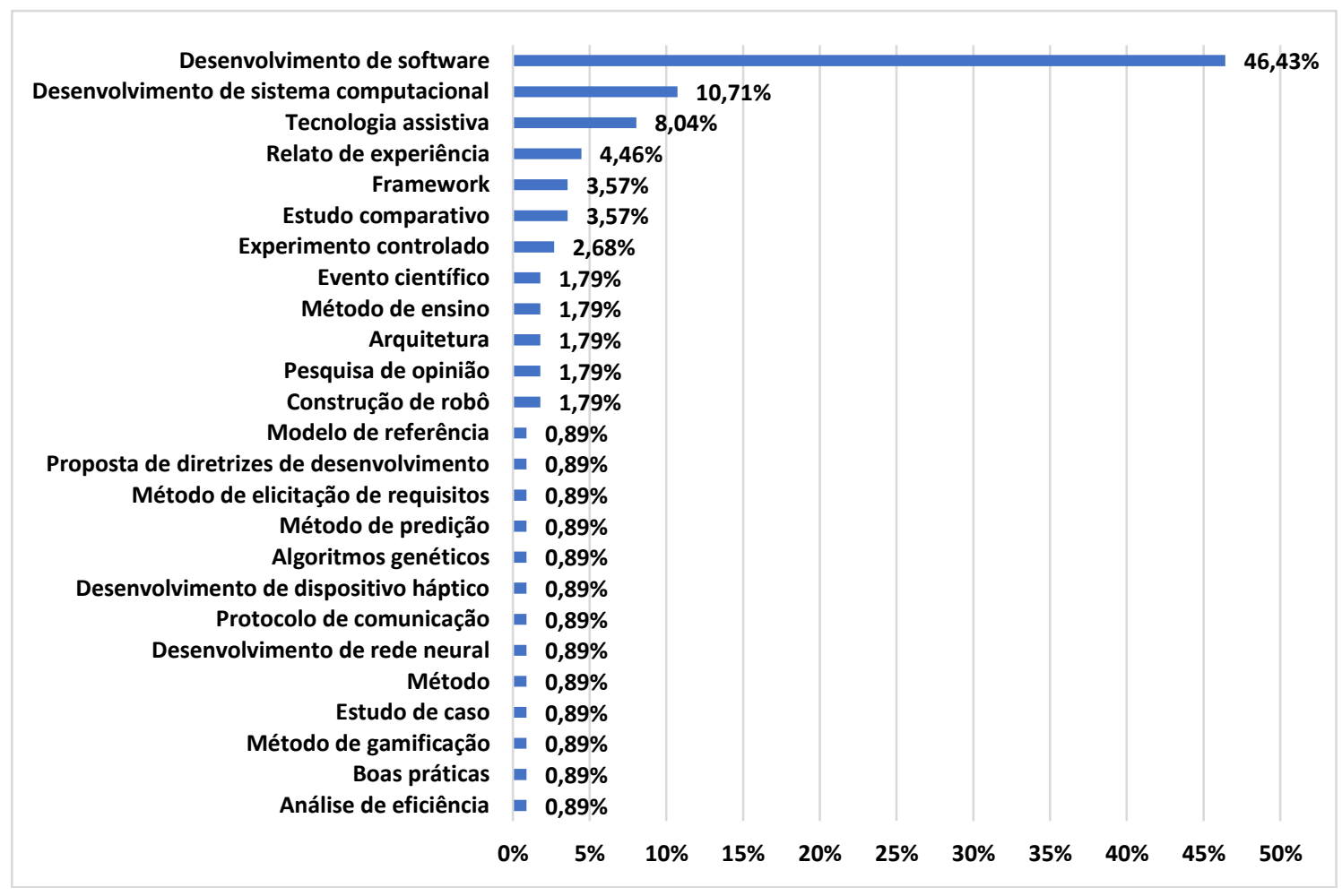

Gráfico 4. Frequência dos tipos de contribuições dos estudos (Fonte: autor)

\subsection{QP4: Os estudos atuam em quais áreas da computação?}

A resposta à esta questão de pesquisa agrupa os estudos em áreas da computação, baseadas nas Comissões Especiais ${ }^{7}$ da SBC. De acordo com o Gráfico 5, a maioria dos estudos atuam

\footnotetext{
${ }^{7}$ https://www.sbc.org.br/403-comissoes-especiais
} 
nas áreas de Informática na Educação (22 estudos), Jogos e Entretenimento Digital (15 estudos), Engenharia de Sistemas Computacionais (14 estudos) e Sistemas de Informação (12 estudos).

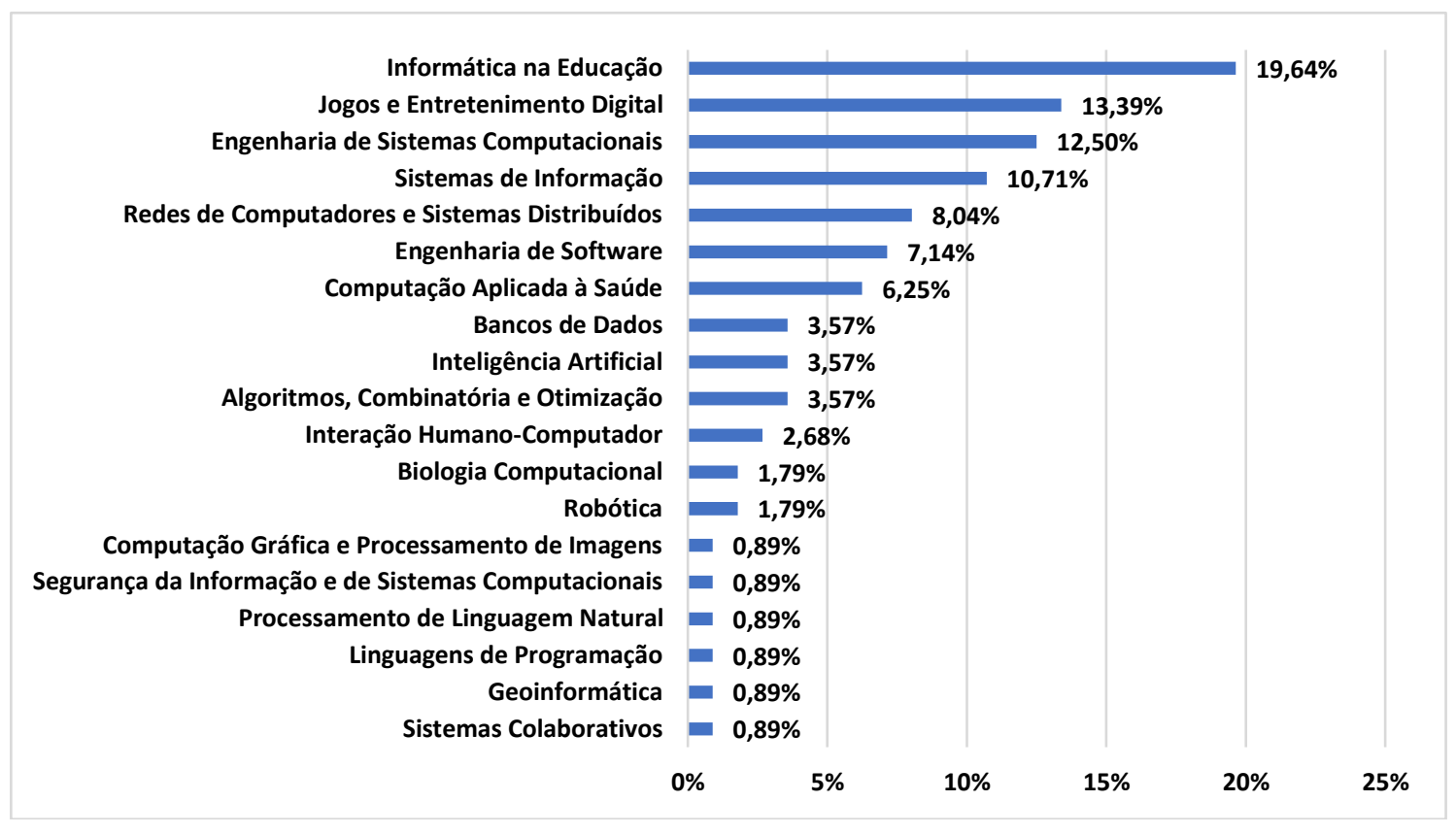

Gráfico 5. Frequência dos estudos por áreas da computação (Fonte: autor)

Os estudos de Informática na Educação em sua maioria, foram estudos que relataram o desenvolvimento de software para apoiar o ensino de diversas áreas, tais como matemática, física, idiomas, lógica de programação, LIBRAS etc. Já a maioria dos estudos classificados como Jogos e Entretenimento Digital são jogos educacionais que apoiam o ensino de várias áreas do conhecimento, como por exemplo, história, física, matemática, biologia, programação, dentre outros.

\section{Considerações Finais}

A computação é uma área que tem sido aplicada em diversos ramos e áreas do conhecimento. Este artigo teve como objetivo apresentar um mapeamento sistemático que permite analisar o estado da arte de pesquisas em computação desenvolvidas em instituições da Rede Federal de ensino. Este estudo secundário possui o contexto de publicações das seis edições do ENCompIF. Através da análise dos dados extraídos dos estudos primários, pode-se observar o histórico de publicações (QP1), os IFs mais atuantes (QP2), as contribuições (QP3) e as áreas de computação mais atuantes (QP4).

Apesar da importância do desenvolvimento de pesquisas em computação, a quantidade de publicações no evento apresentou um pequeno declínio ao longo dos anos. No entanto, observou-se o foco de pesquisas que lançam mão de recursos computacionais para apoiar o ensino de diversas áreas de conhecimento, tais como matemática, programação, ciências, redação de texto, idioma, dentre outros. Paralelamente à Informática na Educação, as pesquisas têm concentrados esforços na área de Jogos e Entretenimento Digital. Neste mapeamento sistemático, todos os estudos primários classificados nesta área são para apoiar o processo de ensino e aprendizagem de algum conteúdo instrucional. Desta forma, os estudos primários classificados como Informática na Educação não adotaram jogos como método de apoio ao ensino. 
Do ponto de vista dos IFs mais atuantes, grande parte tem participado do ENCompIF, destacando-se os estados da Bahia, Mato Grosso do Sul e Santa Catarina.

Como pesquisas futuras, pretende-se realizar outro estudo secundário com o intuito de caracterizar os trabalhos com mais detalhes, como por exemplo, quais perfis de alunos têm participado das pesquisas, quais métodos de validação e análise de dados tem sido adotado.

\section{Referências}

Araujo, R., Fornazin, M., \& Pimentel, M. (2017). Uma Análise sobre a Produção de Conhecimento Científico nas Pesquisas Publicadas nos Primeiros 10 anos da iSys (20082017). iSys-Revista Brasileira de Sistemas de Informação, 10(4), 45-65.

Kitchenham, B. A., Budgen, D. and Pearl Brereton, O. (2011). Using mapping studies as the basis for further research - A participant-observer case study. Information and Software Technology, Special Section: Best papers from the APSEC. v. 53, n. 6, p. 638-651.

MEC. Rede Federal de Educação Profissional, Científica e Tecnológica: Histórico. Disponível em: $<\mathrm{http}: / /$ redefederal.mec.gov.br/historico>. Acesso em: 29 abr 2020.

Petersen, K., Feldt, R., Mujtaba, S. and Mattsson, M. (2008). Systematic Mapping Studies in Software Engineering. Proceedings of the 12th International Conference on Evaluation and Assessment in Software Engineering (EASE'12), p. 68-77.

Silva, M. D., Soares, A. C. B. \& Moura, I. C. (2019). Aplicação de Ferramentas Computacionais para o desenvolvimento do ensino de crianças com autismo: um Mapeamento Sistemático da Literatura. Brazilian Journal of Computers in Education (Revista Brasileira de Informática na Educação - RBIE), 27(3), 351-368. DOI: 10.5753/RBIE.2019.27.03.351.

Souza, D. M., da Silva Batista, M. H., \& Barbosa, E. F. (2016). Problemas e dificuldades no ensino de programação: Um mapeamento sistemático. Revista Brasileira de Informática na Educação, 24(1), 39. 